\title{
Die meistzitierten Arbeiten des 38. Jahrgangs der Psychiatrischen Praxis - Herzlichen Glückwunsch!
}
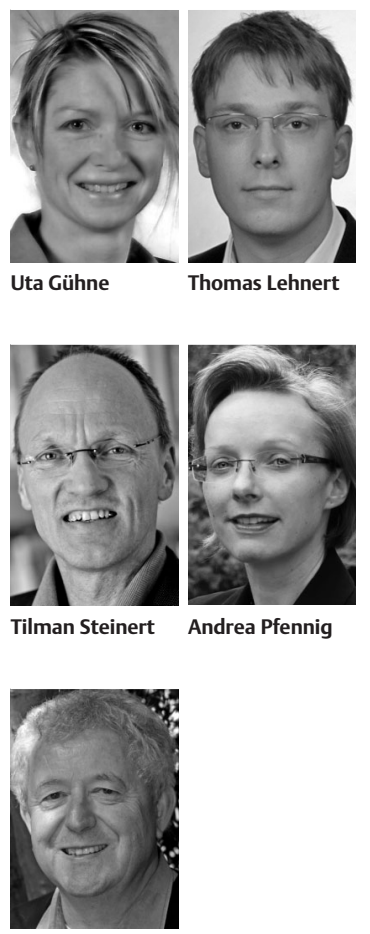

Gerd Hölter

Bibliografie

DOI http://dx.doi.org/ 10.1055/s-0033-1359977

Psychiat Prax 2014; 41: 7

(c) Georg Thieme Verlag KG Stuttgart $\cdot$ New York

ISSN 0303-4259

Korrespondenzadresse

Prof. Dr. med.

Steffi G. Riedel-Heller, MPH Institut für Sozialmedizin,

Arbeitsmedizin und

Public Health (ISAP),

Universität Leipzig

Philipp-Rosenthal-Straße 55

04103 Leipzig

Steffi.Riedel-Heller@medizin.

uni-leipzig.de
Als meistzitierte Arbeit küren wir eine Übersichtsarbeit von Dr. Uta Gühne unter dem Titel: „Akutbehandlung im häuslichen Umfeld - Systematische Übersicht und Implementierungsstand in Deutschland“ [1]. Die Arbeit stellt einen gemeindenahen Versorgungsansatz in den Mittelpunkt, der insbesondere für schwer psychisch kranke Menschen in akuten Krankheitsphasen entwickelt wurde. Die Übersicht zur Effektivität und Kosteneffektivität von Home Treatment wird ergänzt durch die Beschreibung des gegenwärtigen Implementierungsstands in Deutschland. Es wird deutlich, dass Home Treatment eine wirksame Ergänzung bestehender psychiatrischer Angebote darstellt, jedoch in Deutschland bisher wenig etabliert ist. Uta Gühne vom Institut für Sozialmedizin, Arbeitsmedizin und Public Health der Universität Leipzig ist von Haus aus Psychologin und war intensiv an der Entwicklung der S3-Leitlinie Psychosoziale Therapien bei schweren psychischen Störungen beteiligt.

Den zweiten Platz teilen sich eine weitere Übersichtsarbeit und ein kritisches Essay. Diplomsoziologe Thomas Lehnert vom Institut für Versorgungsforschung und Gesundheitsökonomie des Universitätsklinikums Hamburg-Eppendorf legt eine systematische Literaturübersicht zu gesundheitsökonomischen Studien bei Diabetes und komorbider Depression vor [2]. Gleichauf mit der genannten Übersichtsarbeit, sozusagen punktgleich auf dem zweiten Platz, finden wir ein kritisches Essay aus der Feder von Tilman Steinert mit dem Titel „Nach 200 Jahren Psychiatrie: Sind Fixierungen in Deutschland unvermeidlich?“ [3]. Der Beitrag reflektiert das älteste Problem psychiatrischer Institutionen. Er geht der Frage nach, ob es sich bei Fixierungen und anderen Zwangsmaßnahmen um Therapie- oder Sicherheitsmaßnahmen handelt. Darüber hinaus werden die Leser mit einer in Großbritannien praktizierten professionellen Intervention vertraut gemacht, die zur Vermeidung von Fixierungen eingesetzt wird. Prof. Dr. Tilman Steinert, von Haus aus Mediziner, Psychiater, Psychotherapeut und Neurologe, leitet die Abteilung Psychiatrie I der Universität Ulm und ist stellvertretender ärztlicher Direktor und Mitglied der Geschäftsleitung des Zentrums für Psychiatrie in Südwürttemberg. 1997 gründete er den Arbeitskreis der Prävention von Gewalt in der Psychiatrie.

In diesem Jahr wollen wir erstmals auch die meistzitierte Debatte prämieren. Die Prämierung geht an Frau Prof. Dr. Andrea Pfennig und Herrn Prof. Dr. Gerd Hölter. Die Debatte stand unter dem Leitthema „Evidence-Based Medicine ist der Goldstandard der Leitlinienentwicklung“ [4]. Frau Pfennig ist Professorin für psychiatrische Epidemiologie und Verlaufsforschung. Sie ist Psychiaterin und Psychotherapeutin mit einer postgraduierten epidemiologischen Ausbildung und ist an der Klinik für Psychiatrie und Psychotherapie des Universitätsklinikums Carl Gustav Carus der Technischen Universität Dresden tätig. Gerd Hölter ist von Haus aus Philologe und hat den Lehrstuhl für Bewegungserziehung und Bewegungstherapie in Rehabilitation und Pädagogik bei Behinderung an der Fakultät Rehabilitationswissenschaften der Technischen Universität Dortmund inne.

Die prämierten Arbeiten verweisen auf relevante Themen, die weitere Arbeiten stimulierten: Innovative Versorgungsmodelle, die Komorbidität zwischen psychischen und somatischen Erkrankungen [5], Aspekte von Gewalt und Zwang in der Psychiatrie [6] und last not least die evidenzbasierte Medizin und die Leitlinienentwicklung [7].

Wir gratulieren den Autoren und Autorinnen ganz herzlich!

\section{Literatur}

1 Gühne U, Weinmann S, Arnold $K$ et al. Akutbehandlung im häuslichen Umfeld: Systematische Übersicht und Implementierungsstand in Deutschland. Psychiat Prax 2011; 38: 114-122

2 Lehnert T, Konnopka A, Riedel-Heller S et al. Diabetes und komorbide Depression: Systematische Literaturübersicht gesundheitsökonomischer Studien. Psychiat Prax 2011; 38: 369-375

3 Steinert T. Arbeitskreis zur Prävention von Gewalt und Zwang in der Psychiatrie. Nach 200 Jahren Psychiatrie: Sind Fixierungen in Deutschland unvermeidlich? Psychiat Prax 2011; 38: 348-351

4 Pfennig A, Hölter G. Evidence-based Medicine ist der Goldstandard der Leitlinienentwicklung. Psychiat Prax 2011; 38: 218-220

5 Maske UE, Busch MA, Jacobi F et al. Chronische somatische Erkrankungen und Beeinträchtigung der psychischen Gesundheit bei Erwachsenen in Deutschland. Ergebnisse der bevölkerungsrepräsentativen Querschnittsstudie Gesundheit in Deutschland aktuell (GEDA) 2010. Psychiat Prax 2013; 40: 207-213

6 Zinkler M. Neuregelung von Zwang - ein Auftrag für die Fachgesellschaft? Psychiat Prax 2013; 40: 115116

7 Becker T, Riedel-Heller SG. Neue alte Herausforderungen. Psychiat Prax 2012; 39: 365 - 366 